\title{
The Use of ActiGraft, an Autologous Skin Graft, in the Treatment of Complex Diabetes Foot Ulcer - A Case Study
}

\author{
Emre Ozker M. D ${ }^{1}$ and Chinenye Wachuku Meng'* \\ ${ }^{1}$ Acıbadem Altunizade Hastanesi Hospital, Istanbul Turkey \\ ${ }^{2}$ Barry University School of Podiatric Medicine, Miami, Florida
}

Submission: February 01, 2020; Published: February 15, 2020

"Corresponding author: Chinenye Wachuku Meng, Barry University School of Podiatric Medicine, Miami, Florida, USA

\begin{abstract}
Chronic Diabetic foot ulcers (DFUs) are a complication associated with many comorbidities that can result in limb amputation and even death. ActiGraft, an autologous blood clot tissue, showed to be effective in cutaneous hard to heal wounds by promoting the natural healing process of the wound. We present a case of an ActiGraft treatment, applied weekly for 3 weeks on a complicated case of DFU exhibiting extensive necrotic tissue, exposed bone, tendons and fascia. Blood was withdrawn from the patient, at the point-of-care, to create a blood clot that was then placed on the wound. ActiGraft treatment showed promotion of extensive granulation covering the exposed bone and tendon, reduction of necrotic tissue, and decrease in wound area providing a prepared wound bed for skin graft application. ActiGraft was found to be a cost-effective treatment, providing optimal preparation of the wound bed in a complicated case of chronic DFU.
\end{abstract}

Keyword: Autologous; Blood clot; Graft; Wound care; Chronic DFU

Abbreviations: DFU: Diabetic Foot Ulcer; ECM: Extracellular Matrix; ITT: Intent to Treat; PP: Per Protocol

\section{Introduction}

Diabetic foot ulcers (DFUs) are a leading cause of hospitalization in patients with diabetes due to infection, gangrene, amputation, and even death, if necessary, care is not provided [1]. The growing incidence of DFU's creates a huge burden on the medical system with an estimation of an annual cost of over $\$ 10.9$ billion per year in the US [1]. Concerns of healing patients, as well as alleviating burden placed on the medical system, has brought to light the need agents capable of healing complex wounds. ActiGraft, an autologous graft, is used at point-of-care for the safe and rapid preparation of a whole blood clot matrix from a small sample of the patient's own peripheral blood. This creates a biological delivery system, releasing growth factors and cytokines, promoting the natural healing process of the wound. Application of the blood clot tissue serves as a protective covering, biologic scaffold, and an optimal microenvironment [2].

ActiGraft, an autologous fibrin clot, seals surrounding blood vessels that were severed during wound formation and incorporates itself into the tissue, filling in any disruption at the wound site, and initiating hemostasis, the first stage in the healing process. The blood clot then creates a fibrin scaffold, which functions to protect the wound bed by transforming into an interim extracellular matrix (ECM). The newly formed ECM contains necessary cytokines and growth factors that promote tissue repair and progression through the inflammatory stage. While in the proliferation phase, the blood clot functions as a scaffold by recruiting endothelial cells and fibroblasts, in order to construct a permanent ECM. During this phase, the main objective is to continue filling in any disruption in the tissue and close the wound. The blood clot dries out resulting in scab formation. The newly developed scab takes on a protective role by allowing the tissue underneath to remodel without interruption. The current scab falls off, displaying the formation of a collagenous scar [3]. The efficacy of ActiGraft in wound closure by 12 weeks was demonstrated in clinical studies showing healing rates of $64 \%$ and $71.5 \%$ for the intent to treat (ITT) and per protocol (PP) 
populations respectively, and percentage area reduction (PAR) at 4 and 12 weeks of $64 \%$ and $69.2 \%$ for the ITT and PP populations respectively [2,4]. We report here a case study of a hard to heal chronic DFU presenting extensive necrotic tissue, treated with ActiGraft after multiple other therapies failed to manage the wound.

\section{Case Presentation}

An 82-year-old male, with a chronic diabetic foot ulcer on the right foot presented with comorbidities including: Peripheral Arterial Disease, Coronary Artery Disease, and Diabetes Mellitus. The patient was observed with an open wound and abscess localized to the second digit of the right foot with expansion from the second digit down into the midsole of the foot. Previous interventions included balloon angioplasty to the right leg followed by a cross-femoral bypass operation 7 years prior and a right femoro-peroneal bypass operation. The patient experienced an early blockage and underwent another balloon angioplasty to increase blood flow to the distal end of the right lower extremity. The balloon angioplasty extended into the ankle, increasing blood flow, which was supplied by the anterior tibial artery and posterior tibial artery. The patient was admitted to the hospital exhibiting a monophasic arterial doppler and an ankle brachial index of 0.7 for the right leg. He underwent a right second digit amputation as well as an incision, drainage, and debridement of the non-healing ulcer present on the midsole of the foot. Post amputation, necrosis of the tissue was present with no improvement.
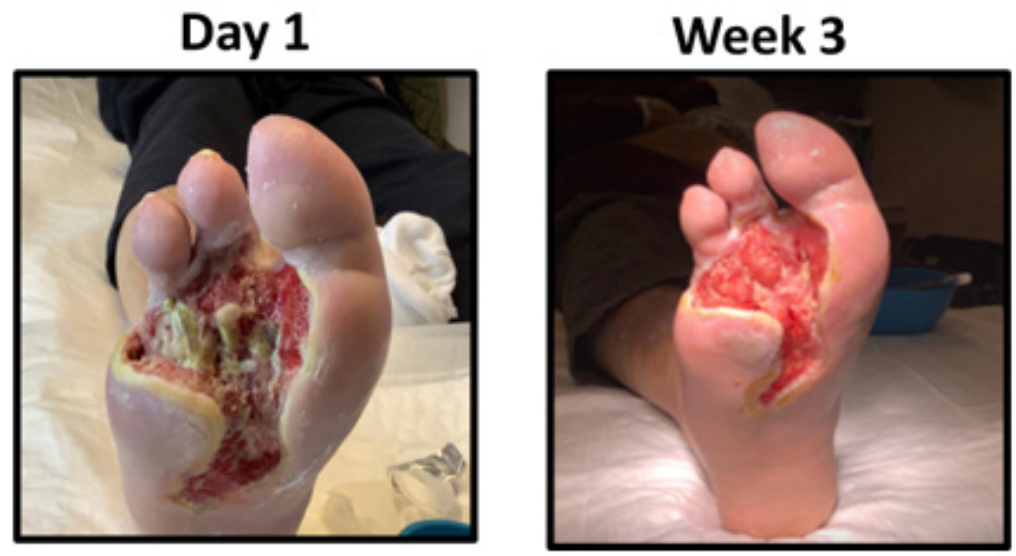

Figure 1: ActiGraft treatment increased granulation growth over the exposed bone, tendons and fascia and reduced the wound area. The wound bed on day 1 shows surrounding hyperkeratotic tissue and edema on the outer-most aspect of the wound periphery with exposed bone, tendon and fascia. Maceration is visible in the inner periphery, progressing distally to the proximal aspect of the 2 nd phalangeal area. On week 3 a massive decrease in necrosis is observed with increase in granulation tissue covering the bone, tendon and fascia. Reduction in wound size was observed.

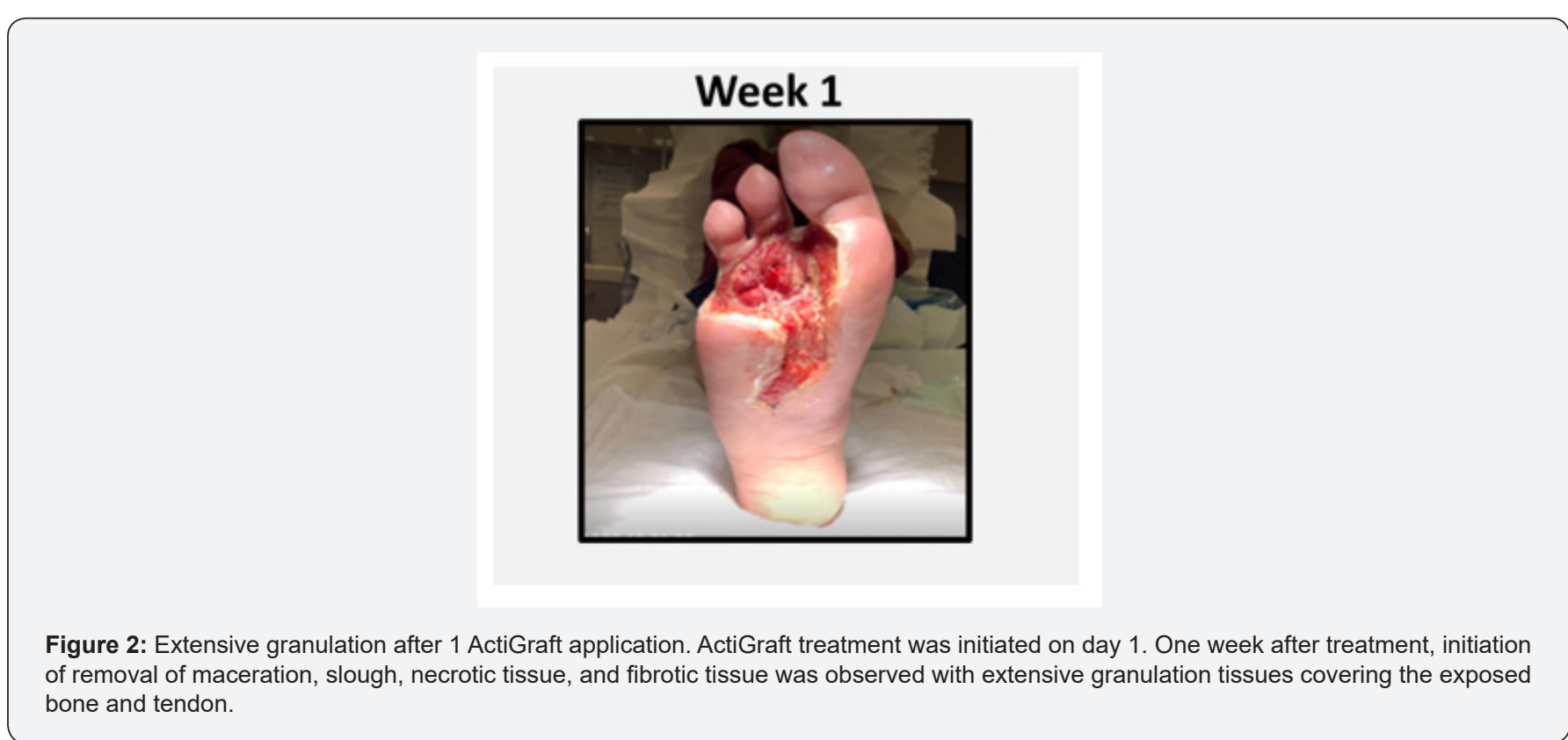


The plantar wound measured $12.6 \mathrm{sq} \mathrm{cm}$. Edema encompassing the midsole of the foot was also observed, extending distally to the tips of the digits. The entire wound edge presented with maceration and hyperkeratotic tissue noted to the wound edge in the midsole. The wound bed consisted of fibrotic tissue, slough, and minimal granulation tissue, consistent with the findings of necrosis. Debridement and dressing changes incorporated with hydrogels and Actolind gel were initiated to manage the condition of the ulcer. At the patient's 2-month post-op visit, necrosis of the tissues had expanded, prompting utilization of larva for treatment. The larva treatment consisted of an application to the wound bed twice a week for 1.5 months with no improvement, resulting in amputation of the right fifth digit. Due to the negative outcome of the larva treatment, the patient was started on a hyperoil treatment for 15 days followed by Abilar twice a day for one month. After all of the undergoing numerous wound care treatment options, the condition of the patient's ulcer showed no improvement, and the wound remained in the inflammatory phase, consistent with a chronic wound exhibiting exposed bone, tendons and fascia (Figure 1).

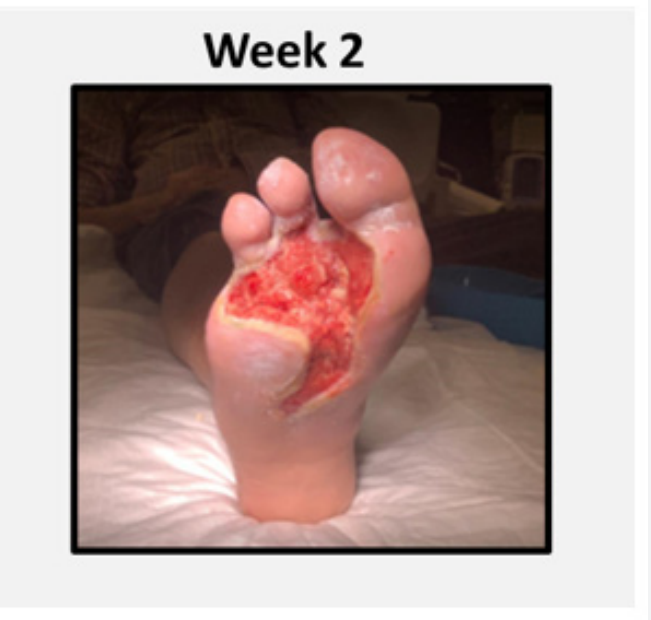

Figure 3: Increased granulation following the 2nd ActiGraft application. Following the second weekly ActiGraft application, an increase in tissue granulation along with reduction in the fibrotic and necrotic tissue were observed.

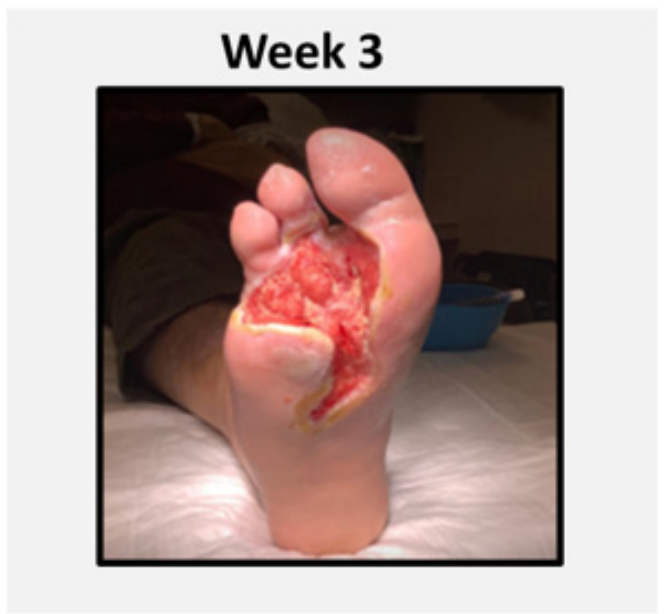

Figure 4: Decrease in wound size following ActiGraft 3rd application. Final application of ActiGraft showed decrease in the wound area with the majority of the macerated tissue revitalized. Increased granulation continued as the necrotic tissue, slough, and fibrotic tissue continued to diminish.

ActiGraft treatment was applied by creating a fibrin clot from the patient's own blood. Treatment was applied weekly on the wound bed, for 3 consecutive weeks (Figure 2-4). On week
3 of ActiGraft treatment, a significant change was observed, demonstrating a massive reduction in maceration, as well as the hyperkeratotic wound edge located at the proximal end of the 
wound. The wound bed had reconciled, exhibiting an extensive amount of granulation tissue, while there was a substantial decrease of slough and fibrotic tissue. The decrease in initial wound size and increase in tissue granulation provided the conditions for further skin graft application (Figure 1).

\section{Discussion}

Chronic DFUs significantly contribute to morbidity and mortality of patients, making the wound difficult to manage leading to increased complications, long-term hospitalization, and gives rise to a risk of undergoing limb amputation [5]. The risk of chronic DFU's deteriorating to the need of amputation was estimated to be 5-8\% within 1 year of treatment despite an aggressive wound management treatment [6], and half of this patient population dies or loses the contralateral limb within 5 years [7], emphasizing the need for new technologies to manage chronic wounds and prevent those outcomes. In this case study, prior to use of ActiGraft, necrosis was present, producing slough and fibrotic tissue in the wound bed. ActiGraft's autologous fibrin scaffold enabled recruitment of necessary factors to repair tissue and develop granulation tissue. With recruitment of macrophages and anti-inflammatory mediators, control of inflammation and infection was achieved. ActiGraft treatment in this complicated case significantly reduced maceration and the hyperkeratotic wound edge, and allowed for a massive decrease in fibrotic tissue, creating the necessary condition for skin graft application to take place. ActiGraft treatment demonstrated rapid tissue granulation growth over the exposed bone, tendons and fascia, with reduction in size of the wound area.

ActiGraft provides an environment in which there is a balance of moisture, enabling proper wound healing. A moist environment has been shown to promote epithelization resulting in reduced scar formation. The inflammatory reaction is then reduced, limiting injury progression [8]. This environment allows for extensive activation of different mediators necessary for mending the soft tissue deficit i.e., cellular migration, proliferation of keratinocytes and fibroblasts, stimulation of growth factors, angiogenesis, collagen synthesis, and coverage of the wound bed. The importance of wound bed preparation by achieving tissue management, inflammation and infection control, moisture balance, and epithelial edge advancement was demonstrated using a weekly application of ActiGraft [9]. ActiGraft being an autologous biodegradable scaffold, mimics the body's natural healing mechanism by replacing the extracellular matrix missing in chronic DFUs, with a fibrin-based matrix created from the patient's own blood at the point of care, reducing the risk of an immunologic rejection $[4,10]$.

\section{Conclusion}

Chronic DFUs commonly present with many factors that inhibit proper wound healing. Extensive comorbidities and complications position the patient in an increased risk for undergoing amputation of the affected limb. In this case study, although countless available approaches were used in attempt to salvage the patient's deteriorating lower extremity, no improvement in terms of vascularization and healing was achieved. ActiGraft treatment prevented limb amputation by initiating the wound healing process in a short amount of time, albeit poor vascularization. With incorporation of the patient's own blood, concerns of possible adverse reactions and further complications were diminished. The newly formed matrix scaffold was able to promote rapid cell ingrowth of granulation tissue through various mechanisms. A weekly ActiGraft application decreased the wound's necrotic tissue and wound size, acting as a wound bed preparatory treatment in a complicated and difficult to heal wound. ActiGraft is a cost-effective measure, alleviating the patient's burden of comorbidities while simultaneously elevating the patient's overall quality of life.

\section{References}

1. Snyder RJ, Hanft JR (2009) Diabetic foot ulcers-effects on QOL, costs, and mortality and the role of standard wound care and advanced-care therapies. Ostomy Wound Manage 55(11): 28-38.

2. Snyder RJ, Schultz G, Wachuku C, Rashid AM, Ead JKK (2020) Proposed Mechanism of Action of Topically Applied Autologous Blood Clot Tissue: A Quintessential Cellular and Tissue Based Therapy. Journal of the American Podiatric Medical Association, pp. 20-140.

3. Donner B, Patel K, Serena Thomas E (2020) Recreating the Natural Wound Healing Environment. Red Dress Medical.

4. Kushnir I, Kushnir A, Garfinkel D, Serena TE (2016) Efficacy and Safety of a Novel Autologous Wound Matrix in the Management of Complicated, Chronic Wounds: A Pilot Study. Wounds Research 28(9): 317-327.

5. Nyamu PN, Otieno CF, Amayo EO, McLigeyo SO (2003) Risk factors and prevalence of diabetic foot ulcers at Kenyatta National Hospital, Nairobi. East African Medical Journal 80(1): 30-43.

6. Kim SY, Kim TH, Choi JY, Kwon YJ, Choi DH, et al. (2018) Predictors for Amputation in Patients with Diabetic Foot Wound. Vasc Specialist Int 34(4): 109-116.

7. Dargis V (1999) Benefits of a multidisciplinary approach in the man-agement of recurrent diabetic foot ulcerations in Lithuania: a prospective study. Diabetes Care 22(9): 1428-1431.

8. Junker JP, Kamel RA, Caterson E, Eriksson E (2013) Clinical Impact Upon Wound Healing and Inflammation in Moist, Wet, and Dry Environments. Advances in Wound Care 2(7): 348-356.

9. Halim AS, Khoo TL, Saad AZM (2012) Wound bed preparation from a clinical perspective. Indian Journal of Plastic Surgery 45(02): 193-202.

10. Snyder RJ, Kasper MA, Patel K, Carter MJ, Kushnir I, et al. (2018) Safety and Efficacy of an Autologous Blood Clot Product in the Management of Texas 1A or 2A Neuropathic Diabetic Foot Ulcers: A Prospective, Multicenter, Open Label Pilot Study. Wounds 30(7): 84-89. 
Your next submission with Juniper Publishers will reach you the below assets

- Quality Editorial service

- Swift Peer Review

- Reprints availability

- E-prints Service

- Manuscript Podcast for convenient understanding

- Global attainment for your research

- Manuscript accessibility in different formats ( Pdf, E-pub, Full Text, Audio)

- Unceasing customer service

Track the below URL for one-step submission https://juniperpublishers.com/online-submission.php 